\title{
Random topologies and the emergence of cooperation: the role of short-cuts
}

\author{
D Vilone ${ }^{1}$, A Sánchez ${ }^{1,2,3}$ and J Gómez-Gardeñes ${ }^{3,4}$ \\ ${ }^{1}$ Grupo Interdisciplinar de Sistemas Complejos (GISC), Departamento de \\ Matemáticas, Universidad Carlos III de Madrid, 28911 Leganés, Madrid, Spain \\ ${ }^{2}$ Instituto de Ciencias Matemáticas CSIC-UAM-UC3M-UCM, \\ 28049 Cantoblanco, Madrid, Spain \\ ${ }^{3}$ Instituto de Biocomputación y Física de Sistemas Complejos, Universidad de \\ Zaragoza, Campus Río Ebro, 50018 Zaragoza, Spain \\ ${ }^{4}$ Departamento de Matemática Aplicada, Universidad Rey Juan Carlos, \\ 28933 Móstoles, Madrid, Spain \\ E-mail: daniele.vilone@gmail.com, anxo@math.uc3m.es and \\ gardenes@gmail.com
}

\begin{abstract}
We study in detail the role of short-cuts in promoting the emergence of cooperation in a network of agents playing the Prisoner's Dilemma game (PDG). We introduce a model whose topology interpolates between the onedimensional Euclidean lattice (a ring) and the complete graph by changing the value of one parameter (the probability $p$ of adding a link between two nodes not already connected in the Euclidean configuration). We show that there is a region of values of $p$ in which cooperation is greatly enhanced, whilst for smaller values of $p$ only a few cooperators are present in the final state, and for $p \rightarrow 1^{-}$cooperation is totally suppressed. We present analytical arguments that provide a very plausible interpretation of the simulation results, thus unveiling the mechanism by which short-cuts contribute to promoting (or suppressing) cooperation.
\end{abstract}

Keywords: game-theory (theory), models for evolution (theory), applications to game theory and mathematical economics, interacting agent models

ArXiv ePrint: 1010.3547 


\section{Contents}

1. Introduction 2

2. The Prisoner's Dilemma game and the evolution of cooperation 3

3. The link added small-world (LASW) network 5

4. The Prisoner's Dilemma game on LASW networks: simulation results 6

5. Discussion 9

5.1. One-dimensional $\operatorname{ring} \ldots \ldots \ldots \ldots$

5.2. The role of short-cuts . . . . . . . . . . . . . . . . . . . 11

6. Conclusions and perspectives $\quad 13$

$\begin{array}{ll}\text { Acknowledgments } & 14\end{array}$

$\begin{array}{ll}\text { References } & 15\end{array}$

\section{Introduction}

Cooperative behaviours are commonly observed in nature and in human society. However, explaining their origin is not a trivial task [1]-[3]. Indeed, at the level of individuals, selfish attitudes are often more convenient (as can be easily seen in many animal behaviours), so there must be some mechanisms that promote a sort of crossover from the 'microscale' level (i.e., in the interactions between a few individuals), to the 'macroscale' one (when a great number of individuals are involved), where cooperation in many cases prevails over defection, reversing the microscopic selfish trend.

Already Nowak and May [4] have suggested that the topology of interactions could be a fundamental factor enhancing the emergence of cooperation even though the interaction is at the individual level. A wealth of studies on evolutionary game theory on graphs were spawned by this first, key insight [5]-[7], making it clear that many factors can favour (or hinder) global cooperative behaviours, for instance the update rule that the individuals use to evolve their strategies (see section 4 below) or the details of the topology; in other words, the outcome of evolutionary games on graphs is far from being universal. From the experimental viewpoint, the situation is similar, and reports that the topology influences [8] and does not influence [9] the emergence of cooperation have been published (although it must be kept in mind that the networks used in those works are very small (see [10]), the maximum size ever tried being $13 \times 13$ [11]). Therefore, it is worth improving our understanding of the roles of both topology and update rules in enhancing (or preventing) cooperation.

Among the topological features that play a key role in evolutionary games, prominent ones are the degree distribution and the clustering. The degree distribution and in particular whether it is of exponential type or has a long tail (scale free in general) has been shown to lead to dramatic differences in behaviour [12,13]. As for the clustering, research indicates that, depending on the specific dynamics, highly clustered networks can promote the survival of initially cooperating agents as they interact mainly among 
themselves, subsequently fostering the spreading of their strategy throughout the whole system $[4,7,14,15]$. This paper is intended to disentangle these effects of clustering from those arising from the existence of short-cuts in the network, which can coexist in many relevant situations. Thus, the Watts-Strogatz (WS) small-world [16] network is the paradigmatic example of local behaviour, like a Euclidean lattice, while acting globally like a random network (and thus having high clustering coefficient and low diameter). Therefore, in this paper we will focus on a model network inspired by the WS one, but with some remarkable differences with respect to it, in order to gain insights into the role of short-cuts in the emergence and sustainability of cooperation.

To this end, the paper has the following structure. In section 2 we summarize the background on the issue that we address here. Subsequently, in section 3 we introduce the model network which we use as the tool of the study, basically a small-world network with additional links. Section 4 presents then our main simulation results, which are followed in section 5 by some theoretical interpretations and implications. Finally, in section 6 we summarize our conclusions and discuss the future perspectives that they open.

\section{The Prisoner's Dilemma game and the evolution of cooperation}

In a general evolutionary framework, $N$ individuals interact through a game. At the initial step every individual plays with a subset of the players and receives a total payoff according to her action and her partners' one. After that, players update their strategy according to an a priori specified update rule. Within this framework, to properly specify an evolutionary game one has to choose a game (defined by the payoffs to the different actions that players can take), an interaction set (with whom every agent plays each time) and a payoff-dependent update rule (how agents modify the strategy governing their actions). Evolutionary game theory on graphs [5] arises when the interaction set is defined as a network: the subset of the players with whom a given one interacts is the set of her neighbours. Thus, in a complete graph all individuals are connected (the well-mixed population case or limit); in a Euclidean square bidimensional lattice every agent interacts with its four nearest neighbours, and so on. On a complex network, the structure can be very complicated and the number of neighbours is in general different for each agent.

The game that we will consider is the Prisoner's Dilemma game (PDG), the paradigmatic model for studying the emergence of cooperation [2]. The PDG is a $2 \times 2$ symmetric game; each player can cooperate $(C)$, or defect $(D)$. If both players cooperate, they get a reward $R$, if both defect they get instead a punishment $P$, and finally if they adopt different strategies the defector earns the temptation payoff $T$, whilst the cooperator receives the sucker's one $S$, with $T>R>P \geq S$. Therefore, the unique Nash equilibrium (a choice of actions for the players from which none of them has incentives to deviate; see, e.g., [17]) is mutual defection, even though this is not a Pareto optimal solution, i.e., there is another configuration, mutual cooperation, in which both players would earn more: here lies the dilemma. Following Nowak and May [4], who first show that cooperation could be enhanced by the presence of a lattice in the PDG, we choose $R=1, T=b \in(1,2]$ and $P=S=0$. This choice (often referred to as the 'weak $\mathrm{PDG}$ ') does not change the essential physics of the model, makes the study easier and reduces to 1 the number of free parameters of the game. While in a well-mixed population (complete graph) a system 
of agents playing the PDG ends up in a frozen state with only defectors [5], [17]-[19] independently of the particular update rule adopted by the agents, when the population structure is a lattice, the system may converge to a final state with a non-vanishing density of survived cooperators $[4,5,7,19,20]$. As mentioned above, subsequent research showed that the effect of the topology is not universal, mainly because it also depends strongly on the update rule adopted by the players [7], [20]-[23] and not only on the particular topology. Below (see section 4) we will consider two update rules in order to assess the scope of our findings.

Regarding the second ingredient of the game, the network, our departure point is the WS one, in its two different versions, WS [16], and Newman-Watts [24] (NW). In the first one each link connecting two nearest neighbours in a unidimensional ring is rewired (that is, one of its two nodes is changed) with probability $p$, whereas in the latter case a short-cut (a new link connecting two originally separated nodes) is added to each site again with probability $p$. Both versions of the model have the property that, according to a parameter $p \in[0,1]$, its topology can be tuned from the Euclidean (for $p=0$ ) to the completely random one $(p=1)$. In the middle, there is a range of values (approximately $0<p \lesssim 0.1$ ) for which the network locally still behaves like a regular lattice, but globally already as a random network $[16,24,25]$. Such models allow us to focus on the effect that the short-cuts in a complex network have over the dynamics and evolution of cooperative behaviours.

To our knowledge, the fact that inserting long-range connections into a regular ring can sensitively influence the evolution and stability of global cooperative behaviours was first noticed in [26]. Subsequently, researchers started studying the specific influence of the decrease of the diameter due to the short-cuts and the onset of heterogeneity in the network (that is, the appearance of hubs created by the added links). Thus, Santos et al [27] showed that in a perfectly homogeneous WS network, cooperation is actually enhanced, but less than in a heterogeneous network. Fu et al [28] reported that there is a peak of the final cooperator density at a certain value of degree heterogeneity, working with a NW network where the short-cuts are added to some fixed hubs in order to have a specified degree of heterogeneity $h$, defined as

$$
h=\frac{1}{N} \sum_{k} k^{2} n(k)-\langle k\rangle^{2}
$$

where $N$ is the number of nodes of the network, $n(k)$ gives the number of vertices with $k$ edges and $\langle k\rangle$ is the average degree. The maximum value of the final cooperation is reached around $h \simeq 0.2$. A similar result was reached by Du et al [29]. Additionally, Vukov et al [30] showed that for homogeneous networks with dynamical noise (that is, with the possibility for an individual to adopt the strategy of worse performing neighbours) longrange connections can diminish the final defector density. Other studies on small-world (or similar) networks, but utilizing different games [31]-[33] or different details of the dynamics, confirmed that short-cuts have a non-trivial and often favourable effect on the emergence of cooperation. On the other hand, on an Erdös-Rényi (ER) network (totally random topology), cooperation is much more enhanced that in both regular lattices and small-world systems [34], a result compatible with the previous ones. In view of all this research, we can now state that in tuning a network from the one-dimensional ring to a totally random network, the cooperation in the final state increases in general, even 
though there can be factors in the dynamics which could sometimes alter this picture. The question then arises naturally as to what occurs if we go instead from the ring topology to the complete graph, in which we know that cooperation is totally suppressed. This is the specific question that we aim at clarifying in this work.

\section{The link added small-world (LASW) network}

Let us now introduce the model for the link added small-world (LASW, in the following) network. The LASW network is defined from a regular one-dimensional ring with $N$ nodes: each site is connected to its $2 m$ nearest neighbours $(m \in \mathbb{N})$, so there are $m N$ links (or edges). The diameter $D_{0}$ (i.e., the average distance between two randomly chosen nodes) and the clustering coefficient $\chi_{0}(\mathrm{~m})$ (i.e., the probability that two neighbours of a third one are also neighbours of each other) of such a simple graph are [25]

$$
\begin{aligned}
& D_{0}=\frac{N}{2 m}, \\
& \chi_{0}(m)=\frac{3(m-1)}{2(2 m-1)} .
\end{aligned}
$$

At this point, we can modify the regular ring by adding new links; that is, we add each of the $[N(N-1) / 2-m N]$ missing edges with probability $p$. Therefore, by varying $p$ we can tune the topology from Euclidean to the complete network as desired. In this model network, it is easy to determine that for this kind of graph the diameter is given by

$$
D=D_{0} f[(N-2 m-1) p],
$$

where the quantity $(N-2 m-1) p=x$ is the density of short-cuts per site and

$$
f(x)= \begin{cases}1 & x \rightarrow 0^{+} \\ \frac{\log (x)}{x} & x \gg 1 .\end{cases}
$$

At the same time, the clustering coefficient is given in the thermodynamical limit by

$$
\chi(p, m)= \begin{cases}\chi_{0}(m) & p=0 \\ p & p \in(0,1] .\end{cases}
$$

Notice how for $p \neq 0$ the clustering coefficient is exactly given by the link-adding probability. The previous expression can be found directly by taking the thermodynamic limit $N \rightarrow+\infty$ in the general result for the clustering coefficient

$$
\chi_{N}(p, m)=z\left[\chi_{0}(m)+\left[1-\chi_{0}(m)\right] p\right]+(1-z) p,
$$

where $z=z(N, m, p)=2 m / \bar{n}(N, m, p)$ is the probability that two nodes are not neighbours in the one-dimensional ring, and $\bar{n}=2 m+p(N-1-2 m)$ is the average number of neighbours per site. We highlight that, except for the case $m=1$, the sequence of functions $\chi_{N}(p, m)$, i.e. the sequence of cluster coefficients given by different values of $N$, does not converge uniformly to the expression for $\chi(p, m)$ given in equation (6), which is, therefore, a non-continuous function in $p$. On the other hand, such pathological behaviour is not alarming, since simulations never deal with really infinite systems. 
It is worth highlighting some remarkable topological features, which will be useful below. First of all, let us consider for simplicity the case $N \gg 2 m+1$, that is $N-2 m-1 \simeq L$. Defining $p^{*}=2 m / N$, we have a crossover in the behaviour of an LASW network. For $p \ll p^{*}$ the number of short-cuts is still much smaller than the number of regular links, and the system behaves similarly to a WS network with rewiring probability $p_{\mathrm{r}}=N p /(2 m)$. In the opposite limit $p \gg p^{*}$, the number of added links is much larger than that of regular ones, and thus the behaviour of the system approaches that of an ER network [35]. Thus, an LASW network is in practice the sum of a regular graph and an ER one, so its degree distribution $P(k)$ is always equal to $P_{\mathrm{ER}}(k+2 m), P_{\mathrm{ER}}(k)$ being the equivalent distribution of an ER network with the same $p$ and $N$ (i.e. a binomial distribution with mean value $p N$ and variance $p(1-p) N)$. In the thermodynamical limit $(N \rightarrow+\infty)$ we have $p^{*} \rightarrow 0^{+}$and the LASW network behaves exactly like an ER one $\forall p \in(0,1]$, with neither its diameter nor its clustering coefficient depending on $m$ (except for $\left.\chi(p=0, m)=\chi_{0}(m)\right)$. Moreover, the clustering coefficient for $m \geq 2$ becomes equal to the value that it has exactly for $m=1$. In practice, for very large networks, the topology is independent of $m$.

\section{The Prisoner's Dilemma game on LASW networks: simulation results}

We now use our model to investigate how the topology influences the emergence of cooperation in a population whose individuals play the PDG. As stated above, we still need to introduce the strategy update rules. Even though there are many possible choices in the literature $[5,7,18]$, we present here only the two most commonly considered ones: unconditional imitation (UI) and the replicator rule (REP). UI is a completely deterministic rule (an automaton): at the end of each round of the game, every player imitates the strategy of the neighbour that has obtained the best payoff provided it is larger that her own payoff. In REP, agents choose a neighbour at random: if the payoff of the chosen neighbour is lower than the agent's own, nothing happens. If it is larger, the agent will adopt the neighbour's strategy with a probability proportional to the difference between the two payoffs. These two rules differ in character (deterministic versus stochastic) and in their outcomes in the presence of a homogeneous network structure: indeed, UI promotes very much the appearance of cooperation whereas REP does not $[4,7,20,21]$. Therefore, while these two rules are by no means the whole panorama of possible dynamics, they will allow us to compare different situations as far as the emergence of cooperation is concerned.

Let us begin from the case of a one-dimensional ring, that is, the case $p=0$, starting from an initial configuration with fifty per cent of cooperators, $m=1$, and UI as the update rule. Numerical simulations show that the final cooperator density is around 0.14 for each value of $b \in(1,2]$ (we will give an analytical explanation for this value in section 5 ). On the other hand, in the opposite limit, $p=1$, the topology corresponds to a fully connected network, and thus there are no cooperators in the final configuration (see section 2). Far from these two limits, highly non-trivial behaviour arises for intermediate values of the link-adding probability $p$. In figure 1 we plot the final density of cooperators on a system with UI dynamics, size $N=1000$, temptation $b=1.05$ and 1.25 , and initial cooperator density equal to 0.5 . The results are really intriguing: for $p \in[0.002,0.2]$ $(b=1.05)$ and for $p \in[0.002,0.02](b=1.25)$ there is a large plateau where cooperation 


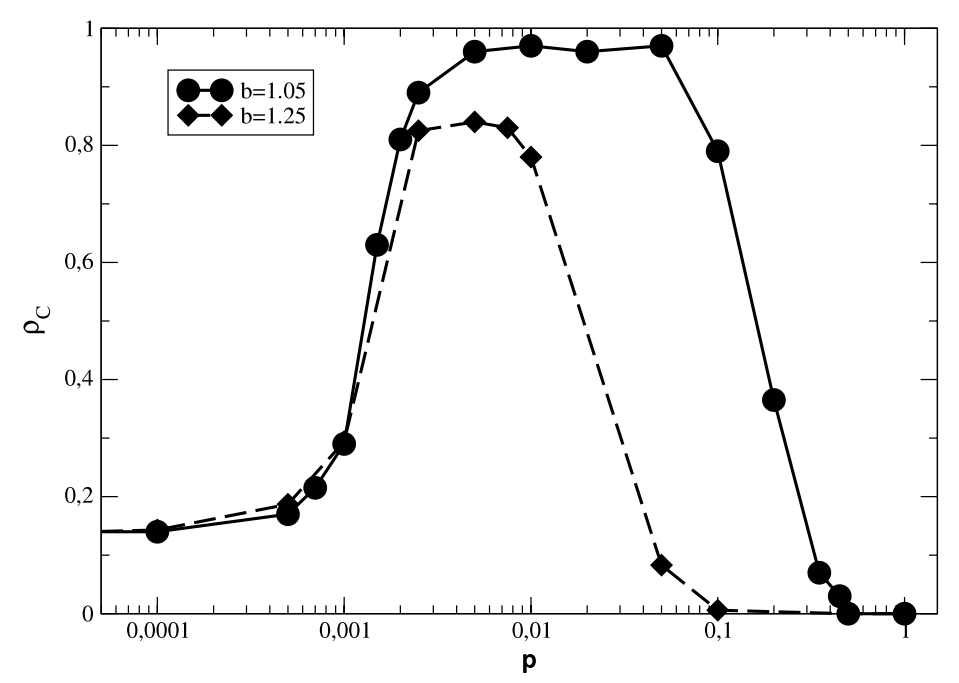

Figure 1. Cooperator density $\rho_{\mathrm{C}}$ (for temptation values of $b=1.05$ (circles) and $b=1.25$ (diamonds)) in the asymptotic state for LASW networks with $m=1$ and $N=1000$ as a function of the link-adding probability $p$. All agents use the UI update rule. Note the logarithmic scale of the $x$ axis.

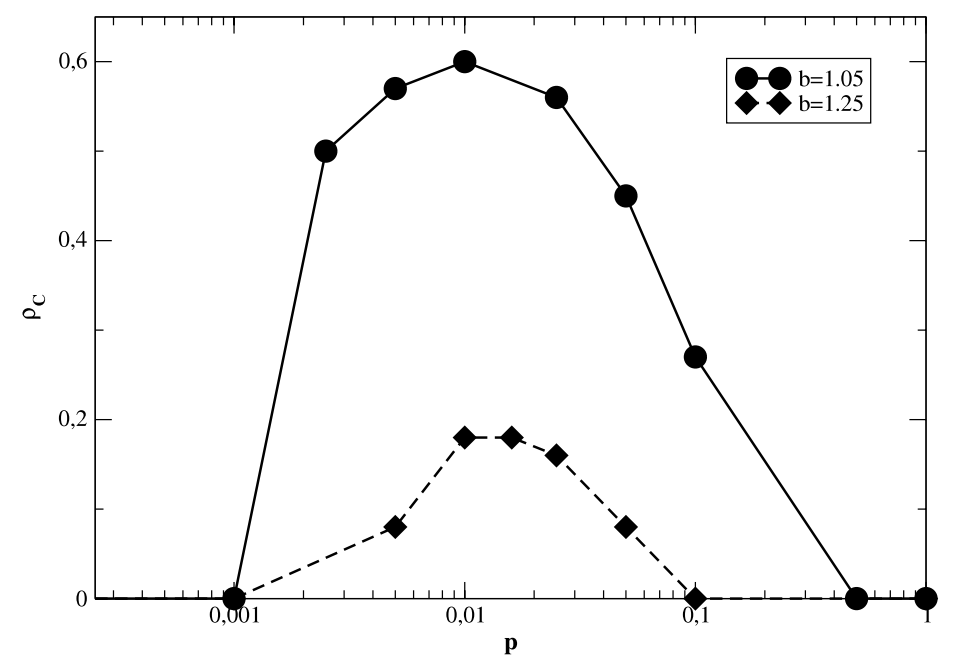

Figure 2. Cooperator density $\rho_{\mathrm{C}}$ (for temptation values of $b=1.05$ (circles) and $b=1.25$ (diamonds)) in the asymptotic state for LASW networks with $m=1$ and $N=400$ as a function of the link-adding probability $p$. All agents use the REP update rule. Note the logarithmic scale of the $x$ axis.

is dramatically enhanced with respect to both the one-dimensional ring $(p=0)$ and, of course, the complete graph $(p=1)$. Such plateaus suggest the existence of an optimal region of the link-adding probability starting at $p \simeq p^{*}$. As observed from figure 1 the length of the optimal region decreases with $b$, as is the case with the cooperator density as well. It is noticeable that the same picture emerges when the update rule used in the system is the REP one, as is clearly shown in figure 2: in this case we also 


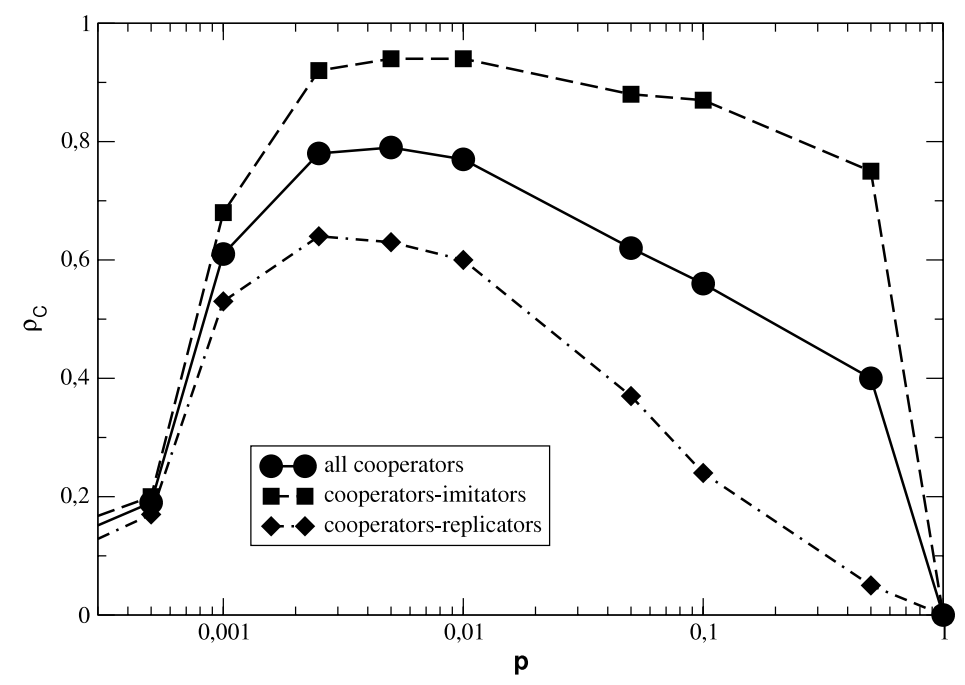

Figure 3. Cooperator density $\rho_{\mathrm{C}}$ (for a temptation value of $b=1.05$ ) in the asymptotic state for LASW networks with $m=1$ and $N=1000$ as a function of the link-adding probability $p$. The system includes the two update strategies (half of the agents use the UI rule, half the REP one). The update rules did not evolve in time.

observe an optimal region of the link-adding probability (starting at $p \simeq p^{*}$ ) in which the cooperation is very great. This means that, apart from the (expected $[7,20]$ ) smaller levels of cooperation in each stage with respect to the UI system, the fundamental mechanism underlying such a phenomenon cannot be due to the update rule chosen, but must be due to the topological features of the network. To further support this claim, we have verified that when update rules coexist in the population, the phenomenology remains practically unchanged, as shown in figure 3. Interestingly, even in such mixed dynamics the UI rule enhances cooperation more than the REP one, consistently with the results presented in the literature mentioned above. Finally, we note also that the cooperation levels of the two fractions of the population are similar to those observed when the corresponding rule is the only one used, providing further evidence of the independence of the results of the update rule considered. It is thus clear that the existence of an optimal range of short-cut density is due to structural features and thus it should be explained in topological terms.

The above results correspond to the case $m=1$, which as explained above we expect to be representative of the evolutionary outcomes for larger values of the initial degree at least for $p>p^{*}$. This is indeed verified in figure 4 , where results for $m=2$ and different systems sizes are shown. Our numerics confirm the existence of a similar transition at $p \simeq p^{*}=2 m / N$, where a plateau of large cooperation densities is observed until this magnitude begins to decrease as $p \rightarrow 1^{-}$. It should be noted, however, that there is an interesting (but not unexpected) difference with respect to the case $m=1$ that arises from the behaviour for $p \rightarrow 0^{-}$. In this limit, the cooperative behaviour in the frozen state is very high but, as we will see in section 5 , this is due to the different geometrical properties of a Euclidean ring with $m \geq 2$. Notwithstanding this special feature, the main conclusion of figure 4 is that for $p \gtrsim p^{*}$ the behaviour of the system is independent of $m$, in the same way as the topology of the system itself. 


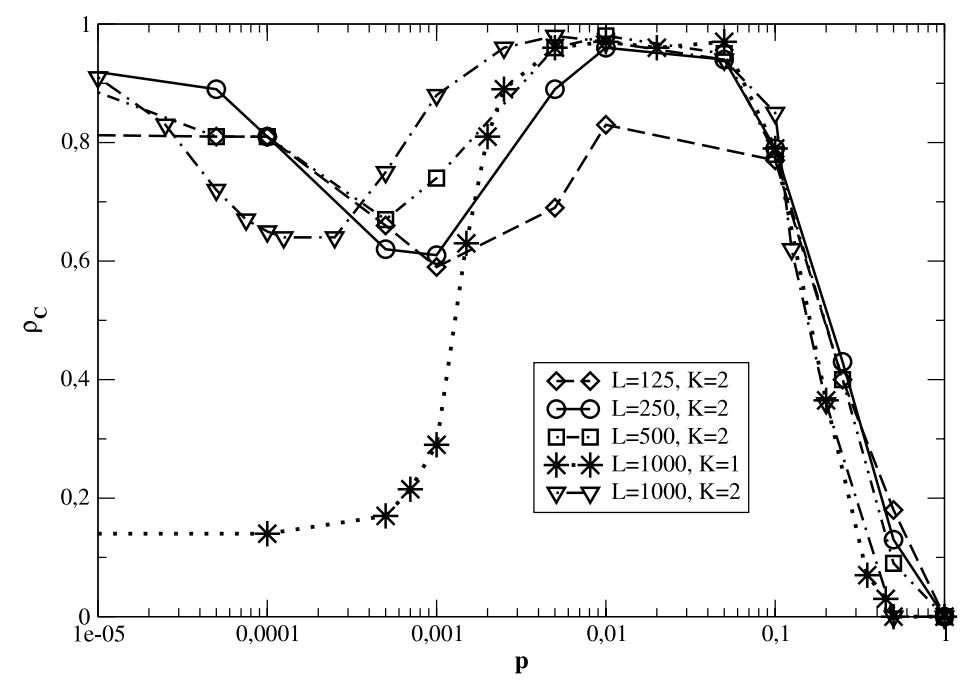

Figure 4. Cooperator density $\rho_{\mathrm{C}}$ (for a temptation value of $b=1.05$ ) in the asymptotic state of LASW networks with $m=2$ and $N=1000$ as a function of the link-adding probability $p$. All agents use the UI update rule. Note the logarithmic scale of the $x$ axis.

Finally, in order to present a more complete picture, we have also analysed the case in which strategies and strategy update rules co-evolve, a line of work that has attracted much interest recently [18]. We will consider the approach proposed in [19], in which agents that decide to copy the strategy of a neighbour copy her strategy update rule as well. Recent results [34] show that such a co-evolutionary process gives rise to different results depending on the topologies and the rules that are initially present in the population. We have therefore carried out simulations with both strategy update rules present in the population in different initial fractions, and allowing them to be adopted as described above. Figure 5 shows that even with evolving update rules, around $p \simeq 0.01$ cooperation is once again promoted, for values up to about $b \simeq 1.4$. This is a further hint of robustness of the cooperation enhancement process due to the short-cuts. We note that, in accordance with the results above, the range in which the cooperation is enhanced decreases with the density of initial replicators. Therefore, all our results show that there are some values of $p$ (and thus of the clustering coefficient - see section 3) for which cooperative behaviour is greatly enhanced regardless of the initial degree of the network and of the evolutionary dynamics. In section 5 we give some theoretical explanations of this phenomenon, which certainly plays an important role in the promotion of cooperation on complex networks.

\section{Discussion}

\subsection{One-dimensional ring}

Let us start our analysis from the simplest case: the one-dimensional ring with $m=1$ (i.e. the case with two neighbours, one left and one right, per site), in which agents play PDG with UI as the microscopical update rule. We will begin by showing that the asymptotic cooperation density does not depend on $b$. Having $b \in(1,2]$, this is an 


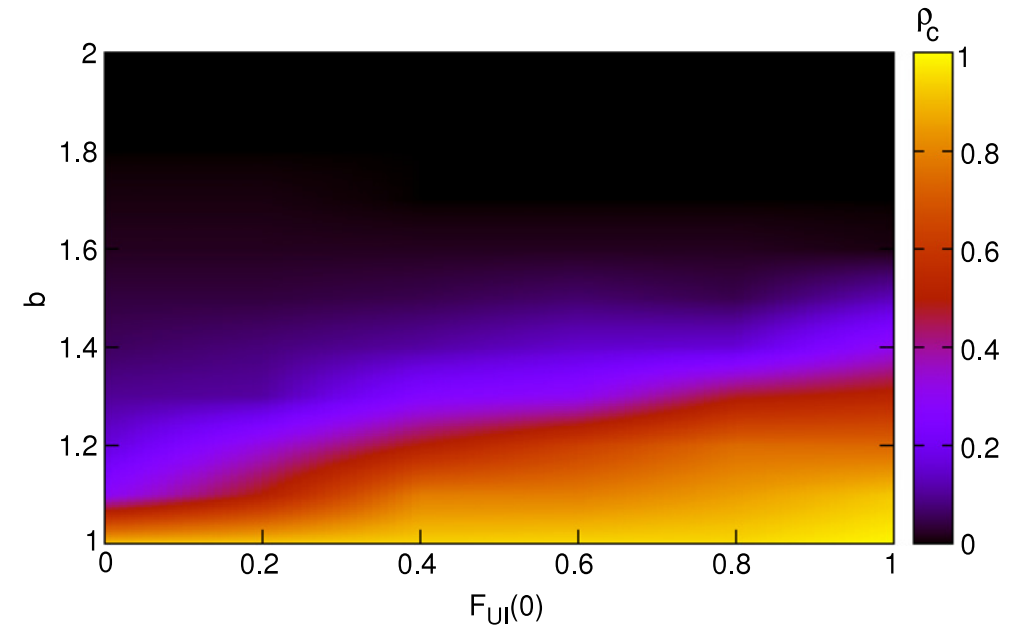

Figure 5. The cooperator density $\rho_{\mathrm{C}}$ in the asymptotic state for LASW networks with $m=1, N=2000$ and $p=0.01$, as a function of the temptation $b$ and of the initial density $F_{\text {ui }}(0)$ of imitators in the initial stage, is shown by means of a colour scale. Update rules (UI and REP) co-evolve with the strategies as described in the text; the initial density of cooperators is equal to 0.5. Note that the larger $F_{\mathrm{ui}}(0)$, the larger the asymptotic level of cooperation.

immediate consequence of the remarkable property of the dynamics that a defector never changes strategy. Indeed, a defector surrounded by at least one cooperator earns from each game a payoff $\Pi \geq b>1$, whilst a cooperator surrounded by at least one defector earns a payoff $\tilde{\Pi} \leq 1<\Pi$, so a $D$-strategist will never find a better performing neighbour with the $C$ strategy. Thus, the evolution of the system is independent of the value of $b$, provided $b>1$. Furthermore, it follows that the cooperator density (that is, the average number of cooperators over the total number of agents) is a decreasing function of time: $\rho_{\mathrm{C}}^{\infty} \leq \rho_{0}$, where $\rho_{\mathrm{C}}^{\infty}=\lim _{t \rightarrow+\infty} \rho_{\mathrm{C}}(t)$ and $\rho_{0}=\rho_{\mathrm{C}}(t=0)$ are the final and initial cooperator densities respectively.

As a second result, we will now show that a necessary condition for a cooperator not to change strategy is to be set in the middle of a cluster of at least three consecutive cooperators. To this end, let us note that a configuration of the form DDCCCDD with cooperators in the boundaries is stable, because the payoffs of the seven players are respectively $b, b, 1,2,1, b, b$. Thus, the defectors and the central cooperator do not have any reason to change, while the cooperators in the boundary of their cluster imitate the central one, and hence they keep the $C$ strategy. Nevertheless, reasoning as above, it must be noticed that a configuration $D C C C D C$ (with cooperators in the boundary) evolves to $D C C D D D$ and finally to $D D D D D D$. In general, isolated defectors give birth to a triplet of $D$-strategists: ...CCCCDCCCC .. goes to ...CCCDDDCCC .., which is a stable configuration.

Having the above considerations in mind, we can say that the final cooperator density must be proportional to the density of clusters of at least three consecutive cooperators in the initial state, minus the effect of the initially isolated defectors $\alpha\left(\rho_{0}\right)$. Then, we can write down

$$
\rho_{\mathrm{C}}=3\left(1-\rho_{0}\right) \rho_{0}^{3}\left(1-\rho_{0}\right)+4\left(1-\rho_{0}\right) \rho_{0}^{4}\left(1-\rho_{0}\right)+\cdots-\alpha\left(\rho_{0}\right),
$$


where each term $l\left(1-\rho_{0}\right) \rho_{0}^{l}\left(1-\rho_{0}\right)$ is the contribution of initial clusters of $l$ cooperators to $\rho_{\mathrm{C}}$ (that is, the number of cooperators $l$ in the cluster times the probability of finding one defector, then $l$ consecutive cooperators, and finally another defector in the initial configuration). Besides, we have $\alpha(x) \in[0,1)$ and $\lim _{x \rightarrow 0^{+}} \alpha(x)=\lim _{x \rightarrow 1^{-}} \alpha(x)=0$. The previous equation can be rewritten as

$$
\rho_{\mathrm{C}}=\rho_{0}^{3}\left(1-\rho_{0}\right)^{2} \sum_{j=0}^{+\infty}(j+3) \rho_{0}^{j}-\alpha\left(\rho_{0}\right)=\rho_{0}^{3}\left(3-2 \rho_{0}\right)-\alpha\left(\rho_{0}\right) .
$$

We stress the fact that the infinite sum in equations (7) and (8) does converge correctly to 1 for $\rho_{0} \rightarrow 1^{-}$, as can be easily seen in the last member of (8).

As in our simulations we always started from $\rho_{0}=0.5$, we have to evaluate the correction term $\alpha\left(\rho_{0}=0.5\right)$. This can be onerous, but the most important correction around $\rho_{0} \simeq 0.5$ is that arising from configurations of the form $\ldots D C C \ldots C C D C C \ldots C C D \ldots$. . . i.e. two clusters of consecutive $C$-strategists being made up by at least three cooperators. Each such initial configuration leads to the removal of the two cooperators surrounding the central defector. In fact the effect is somewhat larger, because some clusters with three cooperators are removed when they are close to a larger cooperator cluster as explained above. Ignoring this effect as a first approximation we can compute, in analogy to the previous calculation,

$$
\alpha\left(\rho_{0}\right) \simeq 3 P_{3}\left(1-\rho_{0}\right) P_{3}=\frac{3 \rho_{0}^{6}}{1-\rho_{0}},
$$

with

$$
P_{3}=\left(1-\rho_{0}\right)^{2}\left(\rho_{0}^{3}+\rho_{0}^{4}+\rho_{0}^{5}+\cdots\right)=\rho_{0}^{3}\left(1-\rho_{0}\right)^{2} \sum_{j=0}^{+\infty} \rho_{0}^{j}=\frac{\rho_{0}^{3}}{1-\rho_{0}} .
$$

Then, in the case of one-dimensional rings with degree equal to 2 and $\rho_{0}=0.5$, from equations (8) and (9) we find $\rho_{\mathrm{C}} \simeq 0.156$, with an $8 \%$ error with respect to the real value of about 0.143 . This good (even though imperfect) agreement shows that we have indeed identified the main mechanisms governing the evolution in the one-dimensional ring.

Note also that the general behaviour described above (cooperator density a decreasing function of time, with the final value only depending on the initial configuration) holds for rules such as UI and REP, where there is no possibility of making mistakes, i.e. one node never copies the strategy of a neighbour with smaller payoff. In this context, it is worth noticing that the model discussed in this subsection is very close to the one described by Eshel et al [36], where mistakes are possible and defectors (called 'egoists' in the paper by Eshel et al) can at some point become cooperators ('altruists').

\subsection{The role of short-cuts}

Let us now consider the case of $p>0$ (always with $m=1$ ). It is easy to see that the presence of short-cuts changes in general the stability of a configuration. In particular, it is no longer true that $\rho_{\mathrm{C}}^{\infty} \leq \rho_{0}$. Indeed, think of a long cluster of defectors, a configuration which is stable in a ring. If we connect one of the internal agents with a far away cooperator surrounded by two other $C$-strategists, at the subsequent step the long-range connected defector will flip toward cooperation. More generally, the fact that a defector can be 
directly connected with more than two cooperators makes the transition $D \rightarrow C$ possible, so $\rho_{\mathrm{C}}^{\infty}$ is expected to increase with $p$ increasing, at least for small values of the link-adding probability. On the other hand, in the limit $p \rightarrow 1^{-}$we know that the cooperation level must vanish [19]. Therefore, we expect a non-trivial behaviour with a maximum of cooperation at some value $\tilde{p} \in(0,1)$. Actually, as shown in figure 1 , in numerical simulations we see a relatively long plateau with a great level of cooperation around $p \sim 0.01$.

Such behaviour can be understood by means of topological considerations. As pointed out in section 3, our network undergoes a sort of (smooth) transition at $p \sim p^{*}=2 k / L$. Indeed, for smaller values of $p$ the system is topologically equivalent to a WS small-world network, whilst for larger values it behaves like an ER one. When we consider the system in figure 1 , we have $p^{*} \simeq 0.002$, and we correspondingly see a sudden increase of the final cooperation just around this value itself. Moreover, in the WS regime the number of short-cuts is not enough yet to affect appreciably the dynamics of the system (there is only a very slight increase of the final cooperators due to the effect described just above). However, when this number becomes large enough (more precisely, when the number of the short-cuts is larger than that of regular links), the presence of several hubs connected with stable cooperators makes the configuration of the system very favourable to the final emergence of the cooperation. Finally, when $p$ approaches 1, the number of short-cuts becomes so large that the system is practically a fully connected graph, hubs are no longer hubs but they are almost like any other node, and the cooperation is completely suppressed [19].

Similar effects happen for $m \geq 2$, but in that case everything is much more complicated. In any event, in the one-dimensional case $(p=0)$ the dynamics is again purely deterministic (with the UI update rule), and the final frozen state depends only on the initial configuration of the system. Given the random initial conditions that we are using, the cooperator density of the frozen state is around 0.96, for large enough system sizes. This means that, for a Euclidean ring, increasing the coordination number enhances the cooperation enormously. This could have been expected because, following the reasoning illustrated above, with four neighbours, a cooperator is likely to be imitated by a defector. For example, let us consider the border between two long clusters, each of opposite strategy (see figure 6 ). Here, provided that $b \in(1,3 / 2)$, the first defector is connected with a cooperator with larger payoff, so she will flip at the next stage to the $C$ strategy (whilst no cooperator is connected with an analogous better-fitting defector). Consequently, the cooperator cluster will increase its size in one unit at each time step, until a frozen state almost completely full of cooperators is reached. If the dynamics is given by the REP rule, the result is the same, although the timescale needed to reach the asymptotic scale is much longer due to the probabilistic nature of the rule.

When short-cuts are added, we have again a different regime, depending on whether $p<p^{*}$ or $p>p^{*}$. In the first regime, there are fewer short-cuts than regular links, and hence the system is topologically like a WS small-world network with $m=2$, i.e. globally it behaves like a random network (very low diameter) whilst locally it resembles a regular lattice (the clustering coefficient almost constant around $\chi_{0}(m=2)=1 / 2$, which decreases, reaching a minimum only for $p \rightarrow p^{*}$ ). When a few short-cuts are present in the system, defection is initially enhanced for the same mechanism as promotes cooperation for $m=1$ : a short-cut could connect a cooperator with a defector with larger payoff, 

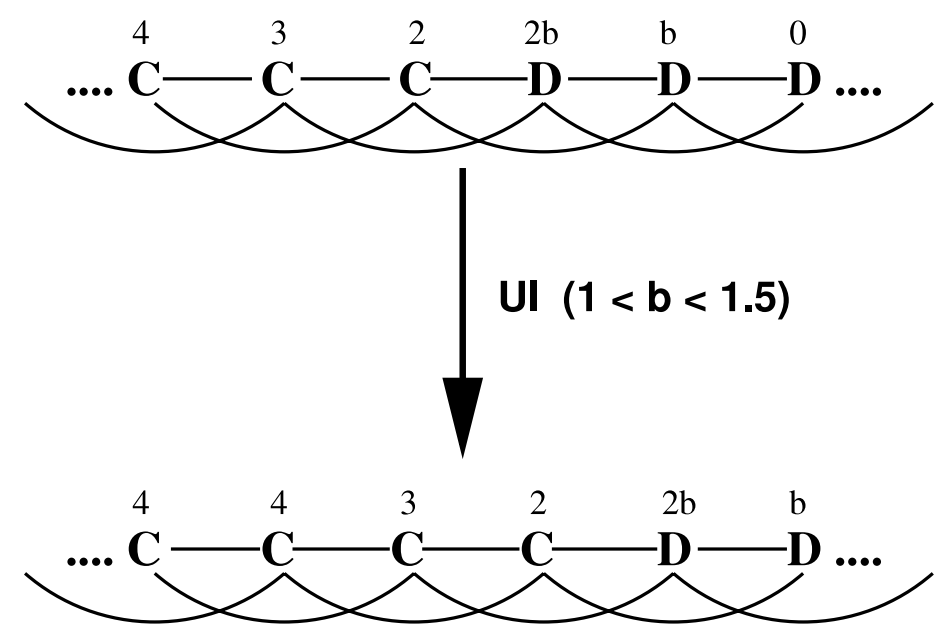

Figure 6. Sample of elementary dynamics on the boundary between two long clusters of different strategy $(m=2)$; the numbers above the individuals (singled out by their strategy) are the payoffs earned after just that time step. With the UI microscopical update rule and $b \in(1,3 / 2)$, the cooperating cluster gains one position at each elementary time step.

driving the first to change her strategy to defection. Finally, once $p>p^{*}$, the system becomes equivalent to an ER random network and the cooperation is enhanced again, until for $p$ large enough we end up with a complete graph where only defectors survive. As can be seen in figure 4 , for each value of $N$, and hence of $p^{*}=2 m / N$, the final level of cooperation decreases when $p \lesssim p^{*}$, then increases rapidly as soon as $p \gtrsim p^{*}$, before vanishing completely for $p \rightarrow 1^{-}$, confirming the interpretation given above.

It must be noticed that all the reasoning and consideration that we have given in this section assumes that the microscopical update rule is the unconditional imitation. We have also indicated along the way that choosing the REP rule will only change the timescale of the evolution. In addition, in so far as there is no possibility of mistakes, the general scheme proposed above also holds with mixed update rules (see figures 3 and 5 ): cooperation is mostly suppressed for $p \lesssim p^{*}$ (WS topology), greatly enhanced for $p \gtrsim p^{*}$ (ER topology), and totally suppressed for $p \rightarrow 1^{-}$(fully connected graph topology). The robustness of such a scheme with respect to the update rules adopted by the individuals confirms the fundamental role of the short-cuts for the ultimate fate of the dynamics.

\section{Conclusions and perspectives}

In this paper we have investigated how the topology of a system in which a population of many individuals interact by means of the PDG can influence the emergence of stable cooperative behaviours, focusing on the effect of short-cuts. For this purpose we exploited a simple model which can assume different topological features of different systems (Euclidean lattice, WS small-world network, ER random network, and fully connected graph) by tuning only the value of a single parameter $p \in[0,1]$ (the linkadding probability). In this way, it is easier to distinguish the precise role that each particular topology plays in enhancing (or suppressing) cooperation. In particular, we 
have shown how a regular Euclidean lattice promotes cooperation only for $m \geq 2, m$ being the coordination number of a one-dimensional ring, while for $m=1$ only a small minority of cooperators survive in the final frozen state. We have also seen that the WS topology does not enhance cooperation, whilst the ER network configuration is the best cooperation promoter of all in this family. Finally, as was already well known, in a fully connected graph (or, equivalently, in the mean-field approximation) only defectors can survive in the final state.

Since the only free parameter of the network model is the link-adding probability $p$ and the results do not depend on the particular dynamics considered, it is the density of short-cuts actually existing in the system that must determine the ultimate fate of the dynamics. Indeed, when $0<p \lesssim p^{*}$, the presence of short-cuts (whose number is still smaller than that of regular links) causes at most a perturbation of the configuration emerging in the Euclidean case (the final level of cooperation is very slightly increased if $m=1$, and not dramatically decreased otherwise), while for $p \gtrsim p^{*}$ in the network many hubs appear, and a cooperator hub is very likely to act as a seed for the spreading of the cooperation throughout the whole system. By means of such a mechanism, the cooperation is enhanced much more than in the other topological regimes. Finally, when $p \rightarrow 1^{-}$, every site ends up being connected to all the others and the system becomes a fully connected graph, where only defectors can survive.

The picture that emerges from our research (that reached the limit of the fully connected network) and from previous works [26]-[30] is that the ER topology may be the best one among homogeneous or mildly heterogeneous graphs for promoting cooperation, and that this property is quite robust, given that the same behaviour (qualitatively) can be found with different update rules (UI and REP dynamics), and also mixing them and letting them co-evolve in the same system. We want to stress that this mechanism for the promotion of cooperation has nothing to do with the one based on clustering discussed in $[7,20]$, as the clustering of the networks where we observe a plateau in the cooperation level is very small $(\sim p)$. This is hence a novel mechanism that has not been discussed before and that confers on short-cuts a very important role in the emergence of cooperation. In fact, they can be used to eliminate the effect of topological traps discussed in [37], which arises due to the existence of bottlenecks and lack of redundant paths. The work summarized here confirms that a not so large fraction of short-cuts can have dramatic effects on the final level of cooperation achieved in the population and clarifies the origin of this mechanism. On the other hand, a more in-depth study of the role of other update rules is still needed; in particular, in the present work we have neglected the family of rules allowing the agents to make some mistakes, i.e., allowing an agent to imitate a worse-fitting neighbour, as for instance happens with the Moran rule $[20,38]$ or with the dynamics in [30]. The case of a two-dimensional initial lattice is also interesting, although we envisage that in that case it will be difficult, if not impossible, to gain insights from an analytical viewpoint as we have done here. Future works should deal with these issues.

\section{Acknowledgments}

DV was supported in part by a postdoctoral contract from Universidad Carlos III de Madrid. AS was supported in part by grants MOSAICO and Complexity-NET RESINEE (Ministerio de Ciencia e Innovación, Spain) and MODELICO-CM (Comunidad de Madrid, 
Spain). JG-G was supported by the MICINN through the Ramón y Cajal programme and grants FIS2008-01240 and MTM2009-13838.

\section{References}

[1] Darwin C, 1871 The Descent of Man (London: John Murray)

[2] Axelrod R and Hamilton W D, 1981 Science 2111390

[3] Maynard-Smith J and Szathmáry E, 1995 The Major Transitions in Evolution (Oxford: Oxford University Press)

[4] Nowak M A and May R M, 1992 Nature 359826

[5] Szabó G and Fáth G, 2007 Phys. Rep. 44697

[6] Tomassini M, Luthi L and Pestelacci E, 2007 Int. J. Mod. Phys. C 181173

[7] Roca C P, Cuesta J A and Sánchez A, 2009 Phys. Life Rev. 6208

[8] Cassar A, 2007 Games Econ. Behav. 58209

[9] Suri S and Watts D J, 2011 PLoS ONE 6 e16836

[10] Helbing D and Yu W, 2010 Proc. Nat. Acad. Sci. 1075265

[11] Grujić J, Fosco C, Araujo L, Cuesta J A and Sánchez A, 2010 PLoS ONE 5 e13749

[12] Santos F C and Pacheco J M, 2005 Phys. Rev. Lett. 95098104

[13] Poncela J, Gómez-Gardeñes J, Campillo M, Floría L M and Moreno Y, 2008 J. Theor. Biol. 253296

[14] Assenza S, Gómez-Gardeñes J and Latora V, 2008 Phys. Rev. E 78017101

[15] Pusch A, Weber S and Porto M, 2008 Phys. Rev. E 77036120

[16] Watts D J and Strogatz S H, 1998 Nature 393440

[17] Hofbauer J and Sigmund K, 1998 Evolutionary Games and Population Dynamics (Cambridge: Cambridge University Press)

[18] Perc M and Szolnoki A, 2010 BioSystems 99109

[19] Moyano L G and Sánchez A, 2009 J. Theor. Biol. 25984

[20] Roca C P, Cuesta J A and Sánchez A, 2009 Phys. Rev. E 80046106

[21] Hauert C and Doebeli M, 2004 Nature 428643

[22] Sysu-Aho M, Saramäki J, Kertész J and Kaski K, 2005 Eur. Phys. J. B 44129

[23] Ohtsuki H and Nowak M A, 2006 Proc. R. Soc. 2732249

[24] Newman M E J, Moore C and Watts D J, 2000 Phys. Rev. Lett. 8432013204

[25] Barrat A and Weigt M, 2000 Eur. Phys. J. B 13547

[26] Masuda N and Aihara K, 2003 Phys. Lett. A 31355

[27] Santos F C, Rodrigues J F and Pacheco J M, 2005 Phys. Rev. E 72056128

[28] Fu F, Liu L H and Wang L, 2007 Eur. Phys. J. B 56367

[29] Du W B, Cao X B, Zao L and Zhou H, 2009 Chin. Phys. Lett. 26058701

[30] Vukov J, Szabó G and Szolnoki A, 2008 Phys. Rev. E 77026109

[31] Wu Z X, Xu X J, Chen Y and Wang Y H, 2005 Phys. Rev. E 71037103

[32] Ren J, Wang W X and Qi F, 2007 Phys. Rev. E 75 045101(R)

[33] Du W B, Cao X B, Yang H X and Hu M B, 2010 Chin. Phys. B 19010204

[34] Cardillo A, Gómez-Gardeñes J, Vilone D and Sánchez A, 2010 New J. Phys. 12103034

[35] Erdős P and Rényi A, On random graphs I, 1959 Publ. Math. Debrecen 6290

[36] Eshel I, Samuelson L and Shaked A, 1998 Am. Econ. Rev. 88157

[37] Roca C P, Lozano S, Arenas A and Sánchez A, 2010 PLoS ONE 5 e15210

[38] Moran P A P, 1962 The Statistical Processes of Evolutionary Theory (Oxford: Oxford University Press) 